\title{
Isolation and characterization of H7N9 viruses from live poultry markets - Implication of the source of current H7N9 infection in humans
}

\author{
SHI JianZhong ${ }^{1 \dagger}$, DENG GuoHua $^{1 \dagger}$, LIU PeiHong $^{2}$, ZHOU JinPing $^{2}$, GUAN LiZheng ${ }^{1}$, \\ LI WenHui ${ }^{1}$, LI XuYong ${ }^{1}$, GUO Jing ${ }^{1}$, WANG GuoJun ${ }^{1}$, FAN Jun ${ }^{1}$, WANG JinLiang ${ }^{1}$, \\ LI YuanYuan ${ }^{1}$, JIANG YongPing $^{1}$, LIU LiLing ${ }^{1}$, TIAN GuoBin ${ }^{1}$, LI ChengJun $^{1 *} \&$ \\ CHEN HuaLan ${ }^{1 *}$
}

\author{
${ }^{1}$ Animal Influenza Laboratory, State Key Laboratory of Veterinary Biotechnology, Harbin Veterinary Research Institute, \\ Chinese Academy of Agricultural Sciences, Harbin 150001, China; \\ ${ }^{2}$ Shanghai Animal Disease Control Center, Shanghai 201103, China
}

Received April 12, 2013; accepted April 15, 2013; published online April 18, 2013

\begin{abstract}
On March 31, 2013, the National Health and Family Planning Commission announced that human infections with a previously undescribed influenza A (H7N9) virus had occurred in Shanghai and Anhui Province, China. To investigate the possible origins of the H7N9 viruses causing these human infections, we collected 970 samples, including drinking water, soil, and cloacal and tracheal swabs of poultry from live poultry markets and poultry farms in Shanghai and Anhui Province. Twenty samples were positive for the H7N9 influenza virus. Notably, all 20 viruses were isolated from samples collected from live poultry markets in Shanghai. Phylogenetic analyses showed that the six internal genes of these novel human H7N9 viruses were derived from avian H9N2 viruses, but the ancestor of their HA and NA genes is uncertain. When we examined the phylogenetic relationship between the H7N9 isolates from live poultry markets and the viruses that caused the human infections, we found that they shared high homology across all eight gene segments. We thus identified the direct avian origin of the H7N9 influenza viruses that caused the human infections. Importantly, we observed that the H7N9 viruses isolated from humans had acquired critical mutations that made them more "human-like". It is therefore imperative to take strong measures to control the spread of H7N9 viruses in birds and humans to prevent further threats to human health.
\end{abstract}

H7N9, influenza virus, human infection, virus source

Citation: Shi J Z, Deng G H, Liu P H, et al. Isolation and characterization of H7N9 viruses from live poultry markets-Implication of the source of current H7N9 infection in humans. Chin Sci Bull, 2013, 58: 1857-1863, doi: 10.1007/s11434-013-5873-4

The influenza A virus genome is composed of eight single-stranded RNA segments that frequently reassort when two different viruses infect the same host [1]. In addition, the RNA-dependent RNA polymerase of influenza virus lacks proofreading ability, which results in a high mutation rate for the viral genome during replication [2]. Mutation and reassortment are the driving forces for the evolution of

$\dagger$ These authors contributed equally to this work.

*Corresponding authors (email: lichengjun@ caas.cn; hlchen1@yahoo.com) influenza viruses, allowing these viruses to reinvent themselves continuously and cause human infections and occasional pandemics.

In March 2013, a previously unidentified novel A (H7N9) influenza virus was isolated from humans in Shanghai and Anhui Province, China. As of April 15, 2013, there have been 63 laboratory-confirmed human cases in six provinces, and 14 of these were fatal. We collected specimens from live poultry markets and poultry farms in Shanghai and Anhui Province and conducted this initial study to charac- 
terize genetically and phylogenetically the novel H7N9 influenza viruses causing human infection and death. We identified H7N9 influenza viruses similar to those causing human infections in birds and environmental samples from the live poultry markets, indicating the direct source of human infection by this new virus.

\section{Materials and methods}

\subsection{Sample collection and virus isolation}

After being informed of the novel cases of human H7N9 infection by the National Health and Family Planning Commission, we conducted active surveillance to identify the origin of the causative viruses. In March 30, 31, 2013, 280 drinking water, soil, cloacal swab, and tracheal swab samples were collected in three live poultry markets in Shanghai (Table 1), and 690 samples from three poultry farms in Shanghai or live poultry markets and poultry farms in Anhui Province. Each sample was placed in $2 \mathrm{~mL}$ minimal essential medium supplemented with penicillin $(2000 \mathrm{U}$ $\left.\mathrm{mL}^{-1}\right)$ and streptomycin $\left(2000 \mathrm{U} \mathrm{mL}^{-1}\right)$. Virus isolation was performed in 10-day-old specific-pathogen-free embryonated chicken eggs. Hemagglutinin (HA) and neuraminidase (NA) subtypes were determined as previously described [3]. All virus isolation procedures were conducted in a biosafety level 3 facility approved by the Ministry of Agriculture, China.

\subsection{Genetic and phylogenetic analysis}

Viral RNA was extracted from virus-infected allantoic fluid with the QIAmp viral RNA mini kit (Qiagen, Hilden, Germany). cDNAs were synthesized from viral RNAs by reverse transcription with Uni12 primer [4], and amplified by polymerase chain reaction with gene-specific primers. The complete genomes for the three H7N9 influenza viruses, A/chicken/Shanghai/S1053/2013 (H7N9), A/environment/ Shanghai/S1088/2013 (H7N9), and A/pigeon/Shanghai/ S1069/2013 (H7N9), were sequenced on an Applied Biosystems DNA analyzer at the Harbin Veterinary Research Institute. Primer sequences are available upon request. The nucleotide sequences were edited using the Seqman module of the DNAStar package. Phylogenetic analysis was performed using the ClustalX 1.81 software package, implementing the neighbor joining method. The tree topology was evaluated by 1000 bootstrap analyses and was shown using Tree View 1.6.6.

\subsection{Nucleotide sequence accession numbers}

The nucleotide sequences of the eight gene segments for the three avian and environmental H7N9 viruses were deposited in the database of Global Initiative on Sharing All Influenza Data (GISAID) under the numbers EPI440678-EPI440701.

\section{Results}

\subsection{Virus isolation}

In total, 20 samples were positive for H7N9 influenza viruses (Table 1). All 20 positive samples were from the 280 samples collected from the three live poultry markets in Shanghai. The other 690 samples collected from the poultry farms in Shanghai, and all of the samples from Anhui Province were negative for virus isolation. Among the 20 positive samples collected from the live poultry markets, ten were isolated from chickens, three from pigeons, and seven from environmental samples.

\subsection{Genetic and phylogenetic analysis}

Prior to the novel H7N9 human infections in China, sequence information was available in the GenBank database for only 25 H7N9 influenza viruses worldwide, and no H7N9 virus has previously been reported in animals or humans in China.

Phylogenetic analysis of the HA gene revealed that the closest relatives in GenBank for the HAs of the three human H7N9 influenza viruses, A/Anhui/1/2013 (H7N9), A/ Shanghai/1/2013 (H7N9), and A/Shanghai/2/2013 (H7N9), were the HAs of four H7N3 influenza viruses isolated from ducks in Zhejiang Province, A/duck/Zhejiang/2/2011 (H7N3), A/duck/Zhejiang/10/2011 (H7N3), A/duck/ Zhejiang/11/2011 (H7N3), and A/duck/Zhejiang/12/2011 (H7N3) (Figure 1(a)). However, they shared only 95.2\%95.8\% nucleotide sequence identity. Therefore, the HA ancestor of these novel H7N9 viruses remains uncertain. In contrast, the three H7N9 viruses isolated in the live poultry markets in Shanghai, A/chicken/Shanghai/S1053/2013 (H7N9), A/environment/Shanghai/S1088/2013 (H7N9), and A/pigeon/Shanghai/S1069/2013 (H7N9), showed 99.1\%99.9\% nucleotide sequence homology with the three H7N9 human isolates (Table 2). We therefore established that the HA gene of the novel H7N9 human viruses was directly acquired from similar H7N9 viruses circulating simultaneously in avian hosts and the environment.

The NA genes of the three human H7N9 viruses shared

Table 1 Information on samples collected from Shanghai live poultry markets and tested for H7N9 influenza viruses ${ }^{\text {a) }}$

\begin{tabular}{lcc}
\hline \multicolumn{1}{c}{ Source of samples } & $\begin{array}{c}\text { Total number } \\
\text { of samples }\end{array}$ & $\begin{array}{c}\text { H7N9 virus-positive } \\
\text { samples }\end{array}$ \\
\hline Chickens & 150 & 10 \\
Pigeons & 35 & 3 \\
Ducks & 30 & 0 \\
Environment $^{\text {b) }}$ & 65 & 7 \\
\hline
\end{tabular}

a) A total of 970 samples were collected from poultry farms and live poultry markets in Shanghai and Anhui Province. Samples collected from Anhui Province and from farms in Shanghai were H7N9-negative and therefore not included in the Table. b) Soil, water, and fecal samples were collected from different areas of the markets. 
(a) HA

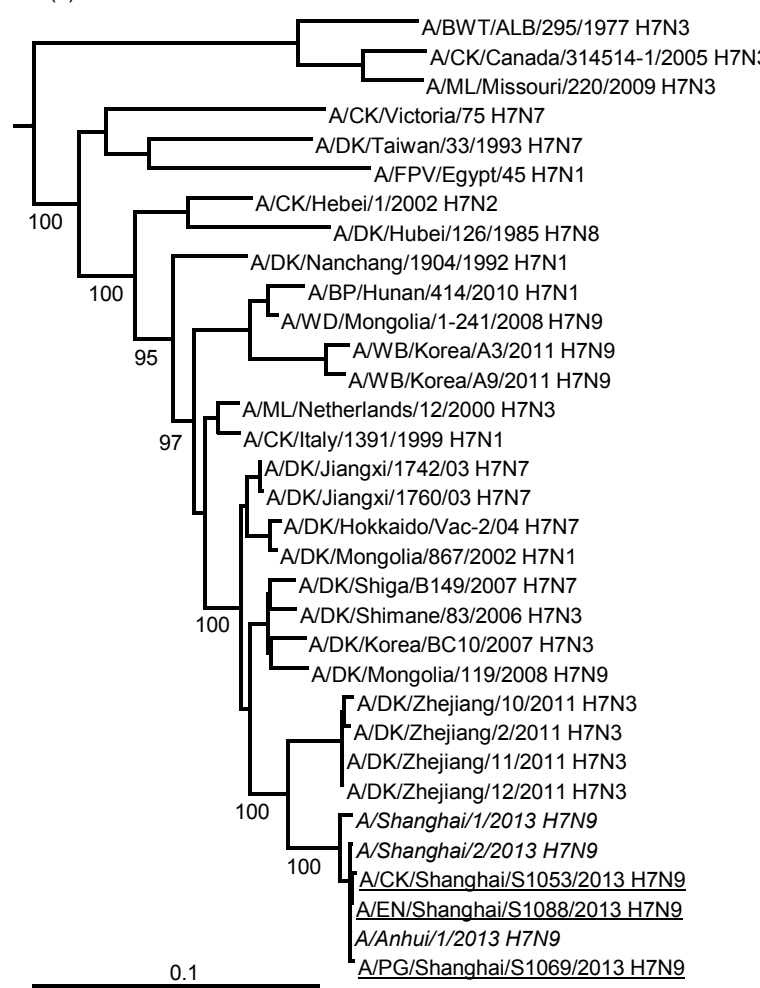

(b) NA

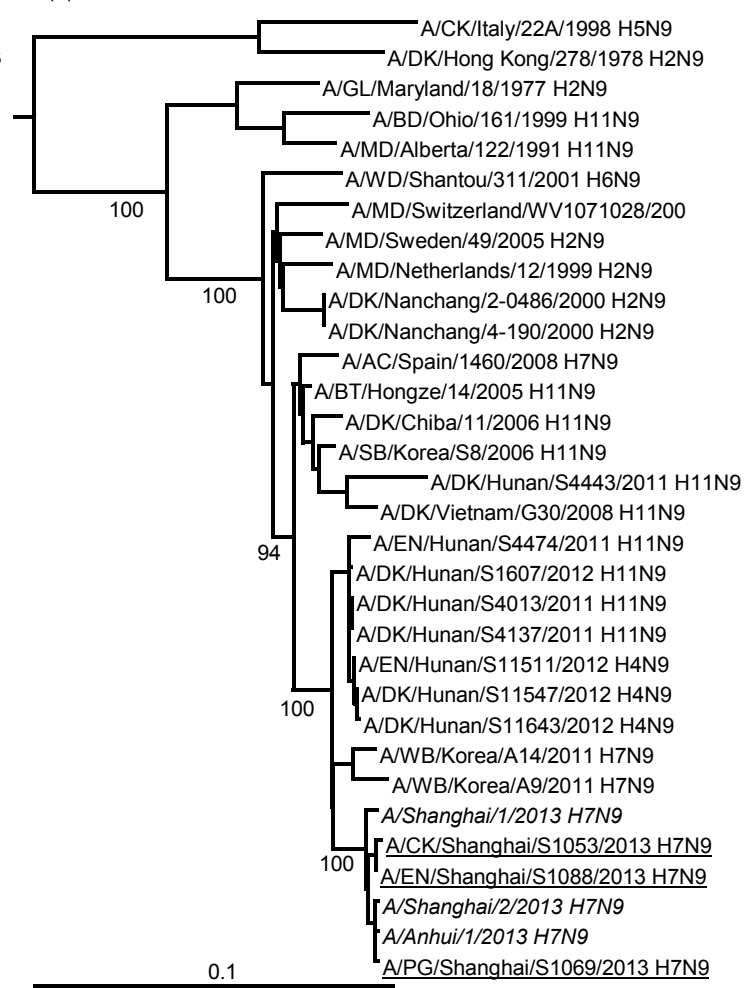

Figure 1 Phylogenetic analyses of the HA (a) and NA (b) genes of the novel H7N9 influenza viruses. Phylogenetic analysis was performed using ClustalX 1.81 software, implementing the neighbor joining method. The tree topology was evaluated by 1000 bootstrap analyses and shown using Tree View 1.6.6. Neighbor-joining bootstrap values $\geqslant 70$ were shown at the major nodes of the phylogenetic trees. The H7 HA tree was rooted to A/EQ/Prague/1/56 (H7N7) and the N7 NA tree to A/KG/Argentina/LDC4/2006 (H13N9). The regions of the nucleotide sequences used for the phylogenetic analysis were: HA, 76-1693 and NA, 35-1408. Viruses characterized in the present study were underlined in the HA and NA phylogenetic trees. The HA and NA sequences for H7N9 viruses causing human infections were downloaded from the database of Global Initiative on Sharing All Influenza Data (GISAID) and were shown in italic. AC, Anas crecca; BD, black duck; BL, brambling; BP, baer'spochard; BT, baikal teal; BWT, blue-winged teal; CK, chicken; DK, duck; EN, environment; EQ, equine; GL, gull; GS, goose; KG, kelp gull; MD, mallard duck; ML, mallard; PG, pigeon; SB, shorebird; WB, wild bird; WD, wild duck.

Table 2 Genome sequence similarity among the H7N9 viruses isolated from live poultry markets and from humans

\begin{tabular}{|c|c|c|c|c|c|c|c|c|c|}
\hline \multirow{3}{*}{ Gene } & \multicolumn{9}{|c|}{ Nucleotide sequence homology $(\%)^{\text {a) }}$} \\
\hline & \multicolumn{3}{|c|}{ A/pigeon/Shanghai/S1069/2013(H7N9) } & \multicolumn{3}{|c|}{ A/chicken/Shanghai/S1053/2013(H7N9) } & \multicolumn{3}{|c|}{ A/environment/Shanghai/S1088/2013(H7N9) } \\
\hline & $\begin{array}{c}\text { A/Shanghai/ } \\
1 / 2013 \\
\end{array}$ & $\begin{array}{c}\text { A/Shanghai/ } \\
2 / 2013 \\
\end{array}$ & $\begin{array}{c}\text { A/Anhui/ } \\
1 / 2013\end{array}$ & $\begin{array}{c}\text { A/Shanghai/ } \\
1 / 2013 \\
\end{array}$ & $\begin{array}{c}\text { A/Shanghai/ } \\
2 / 2013 \\
\end{array}$ & $\begin{array}{c}\text { A/Anhui/ } \\
1 / 2013\end{array}$ & $\begin{array}{c}\text { A/Shanghai/ } \\
1 / 2013 \\
\end{array}$ & $\begin{array}{c}\text { A/Shanghai/ } \\
2 / 2013\end{array}$ & $\begin{array}{c}\text { A/Anhui/ } \\
1 / 2013\end{array}$ \\
\hline HA & 99.1 & 99.8 & 99.9 & 99.1 & 99.8 & 99.8 & 99.2 & 99.9 & 99.9 \\
\hline NA & 99.3 & 99.9 & 99.9 & 99.3 & 99.3 & 99.3 & 99.4 & 99.5 & 99.5 \\
\hline PB2 & 99.5 & 99.8 & 99.9 & 99.6 & 99.8 & 99.9 & 99.6 & 99.9 & 100 \\
\hline PB1 & 96.5 & 96.7 & 96.8 & 99.4 & 99.9 & 99.9 & 99.4 & 100 & 100 \\
\hline PA & 99.8 & 99.9 & 99.9 & 99.9 & 100 & 100 & 99.6 & 99.8 & 99.7 \\
\hline NP & 97.7 & 98.8 & 98.8 & 97.6 & 100 & 100 & 97.6 & 100 & 100 \\
\hline M & 99.9 & 99.9 & 99.9 & 99.9 & 99.9 & 99.9 & 99.9 & 99.9 & 99.9 \\
\hline NS & 97.7 & 100 & 100 & 97.7 & 100 & 100 & 97.7 & 100 & 100 \\
\hline
\end{tabular}

a) Analysis was based on the sequence of HA, 76-1693; NA, 35-1408; PB2, 58-2283; PB1, 48-2230; PA, 25-2029; NP, 46-1523; M, 26-784; NS, 27-719.

the highest nucleotide sequence homology (97.2\%-97.3\%) with the NA gene of the A/wild bird/Korea/A14/2011 (H7N9) virus from GenBank. During our active surveillance of avian influenza viruses in domestic ducks at Dongting Lake, China, we isolated three H4N9 and five H11N9 vi- ruses from ducks or duck farm environment (unpublished data). The N9 NA genes of these eight viruses, with the exception of A/duck/Hunan/S4443/2011 (H11N9), shared $97.3 \%-97.9 \%$ homology with the NA genes of the three H7N9 human viruses. There was a deletion of five amino 
acids at positions 69-73 in the NA gene of the human H7N9 viruses, which was not present in the Dongting Lake H4N9 or H11N9 viruses or in the A/wild bird/Korea/A14/2011 (H7N9) virus. It is therefore, unclear whether the novel H7N9 viruses derived their NA gene from the Dongting Lake viruses or Korean H7N9 virus. As with the HA gene, the NA genes of the three H7N9 avian and environmental viruses were the direct sources for the NA genes of the human H7N9 viruses, sharing 99.3\%-99.9\% nucleotide sequence homology (Figure 1(b) and Table 2).

The PB2 genes of the three H7N9 human viruses were clustered with the three avian and environmental H7N9 viruses and shared 99.5\%-100\% homology (Figure 2(a) and Table 2). This high similarity was also seen for the PA gene, which showed $99.6 \%-100 \%$ nucleotide sequence homology between the human and avian or environmental H7N9 viruses (Figure 2(c) and Table 2). The PB1 genes of the three H7N9 avian and environmental viruses were present in two forms (Figure 2(b)). The PB1 gene of A/pigeon/Shanghai/ S1069/2013 (H7N9) virus shared 96.5\%-96.8\% homology with the PB1 genes of the three human H7N9 viruses, whereas the PB1 genes of the H7N9 chicken and environmental viruses shared $99.4 \%-100 \%$ nucleotide sequence homology with the three human H7N9 viruses (Table 2). Furthermore, we found that an H9N2 virus, A/brambling/ Beijing/16/2012 (H9N2), was the closest relative in GenBank of the novel H7N9 viruses with respect to all three polymerase genes (Figure 2(a)-(c)). The homology between the A/brambling/Beijing/16/2012 (H9N2) virus and the three human $\mathrm{H} 7 \mathrm{~N} 9$ viruses for the PB2, PB1, and PA genes was $99.1 \%-99.2 \%, 98.3 \%-98.7 \%$, and $99.2 \%-99.3 \%$, respectively. In addition to A/brambling/Beijing/16/2012 (H9N2) virus, the three polymerase genes of many other H9N2 viruses in GenBank were similar to those of the H7N9 human viruses. This indicates that the human H7N9 viruses derived their three polymerase genes from avian H9N2 viruses.

The NP gene of A/Shanghai/1/2013 (H7N9) virus was $97.6 \%$ homologous to the other two human H7N9 viruses, A/Anhui/1/2013 (H7N9) and A/Shanghai/2/2013 (H7N9), which showed $100 \%$ homology with each other. The NP genes of the three H7N9 avian and environmental viruses were closely related to those of the A/Anhui/1/2013 (H7N9) and A/Shanghai/2/2013 (H7N9) human viruses with 98.8\%-100\% identity (Figure 2(d) and Table 2). The $M$ and NS genes of the human and avian or environmental H7N9 viruses were highly similar with $99.7 \%-100 \%$ homology (Figure 2(e), (f) and Table 2). In addition to the avian and environmental H7N9 viruses, many H9N2 viruses in GenBank also clustered with the novel H7N9 viruses in the phylogenetic trees of these three genes. This suggests that the NP, M and NS genes of the H7N9 viruses are derived from H9N2 viruses. Therefore, the H9N2 viruses were the ancestors for all six of the internal genes of the novel H7N9 viruses that have infected humans and are currently circu- lating in avian species and the environment of live poultry markets in Shanghai.

\subsection{Molecular signatures of the novel H7N9 viruses}

The HA gene of the three human H7N9 viruses and the avian and environmental H7N9 viruses in this study lacked the stretch of multi-basic amino acids at the HA cleavage site, which is the signature of highly pathogenic $\mathrm{H} 5$ and $\mathrm{H} 7$ influenza viruses [5,6]. However, human infections with this H7N9 virus still cause severe symptoms and a considerably high fatality rate. This suggests that these H7N9 viruses can replicate in humans efficiently, even though their HA is considered to be low-pathogenic. HA receptor-binding specificity is a major molecular determinant of host range for influenza viruses. The amino acids at positions 226 and 228 of HA are critical for the receptor-binding specificity of influenza virus [7-9], and the mutations Q226L and G228S in HA increase the binding affinity of H7N7 viruses to human-type $\alpha-2,6$ sialylated glycans [10]. Two of the human H7N9 viruses, A/Anhui/1/2013 (H7N9) and A/Shanghai/2/2013 (H7N9), and the three avian and environmental H7N9 viruses had "human-like" residue leucine at position 226, which implies that these viruses may have partially acquired human-type receptor-binding specificity.

The $627 \mathrm{~K}$ and $701 \mathrm{~N}$ residues in the PB2 protein contribute to the replication and transmission of avian influenza viruses in mammalian hosts [11-14]. The three avian and environmental H7N9 viruses had the avian-like 627E residue. In contrast, all three human H7N9 viruses have acquired the $627 \mathrm{~K}$ mutation in their PB2 protein, which may have significantly contributed to their pathogenicity and lethality in humans. The NS1 protein of the novel H7N9 viruses is truncated by a stop codon at position 218 , which creates a deletion of the PDZ-binding domain, a protein-protein interaction domain that has been implicated in the pathogenicity of the 1918 virus and highly pathogenic H5N1 viruses [15]. The PB1-F2 protein has been shown to be related to the increased pathogenicity of the 1918 virus and highly pathogenic H5N1 viruses [16,17]. With the exception of the PB1-F2 protein of the A/pigeon/Shanghai/ S1069/2013 (H7N9), which was truncated by a stop codon at position 26, the other five human and avian or environmental H7N9 viruses all encoded the full-length PB1-F2 protein. In addition, the amino acid sequence of the M2 protein of the six human, avian, and environmental H7N9 viruses contained the $\mathrm{S} 31 \mathrm{~N}$ substitution, a characteristic of amantadine resistance in influenza viruses $[18,19]$.

\section{Discussion}

We showed that similar H7N9 viruses were simultaneously prevalent in chickens, pigeons, and the environment in the 
(a) PB2

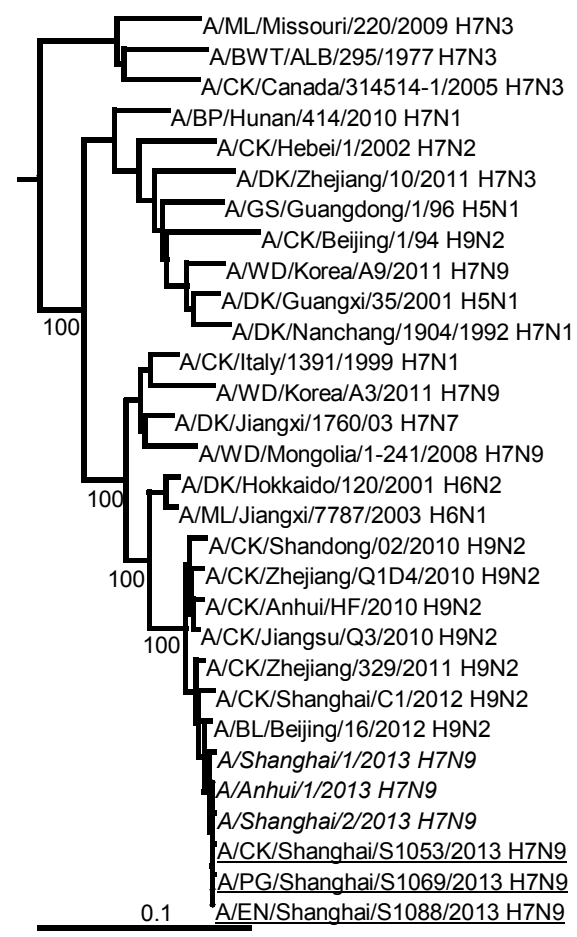

(d) NP

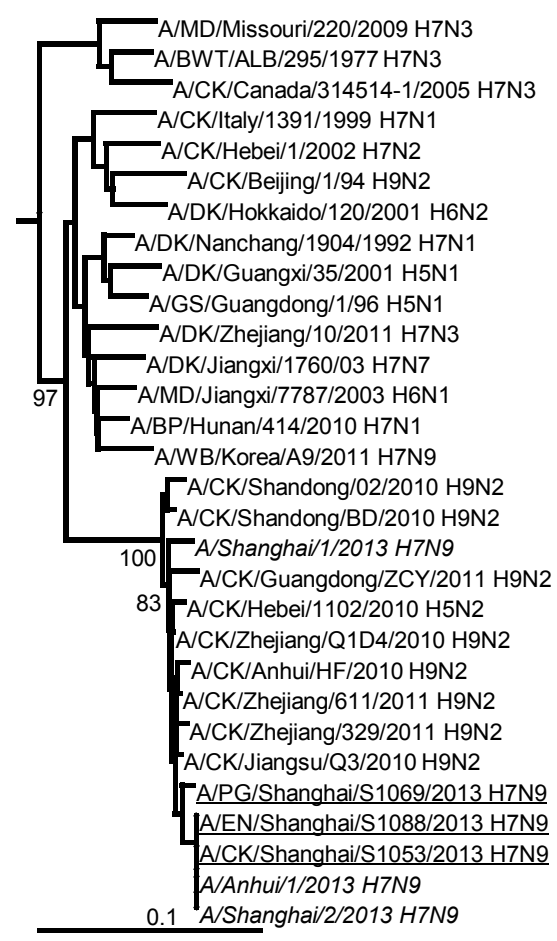

(b) PB1

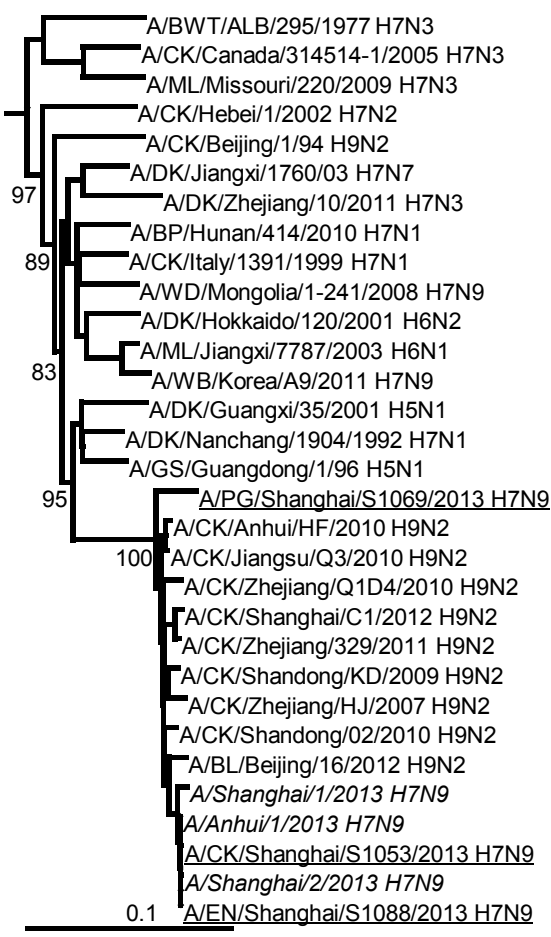

(e) $\mathrm{M}$

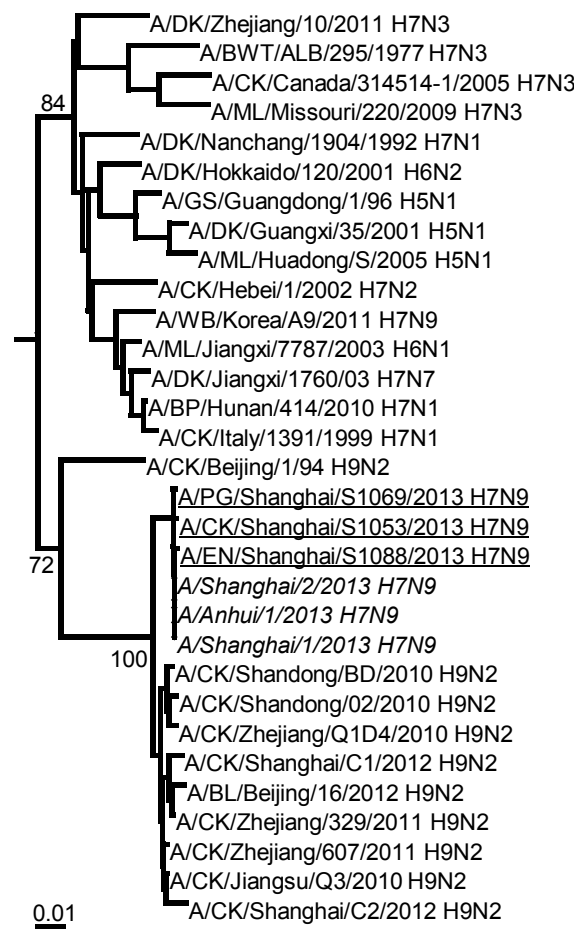

(c) PA

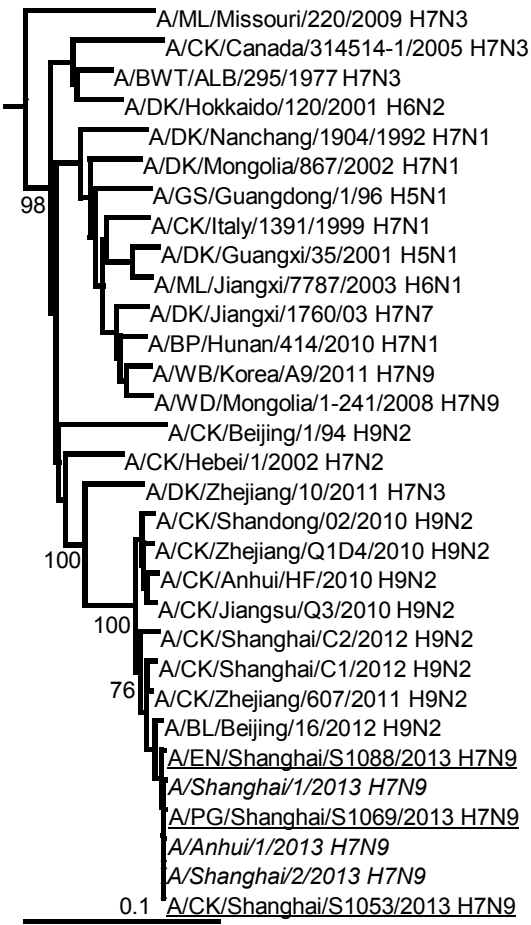

(f) NS

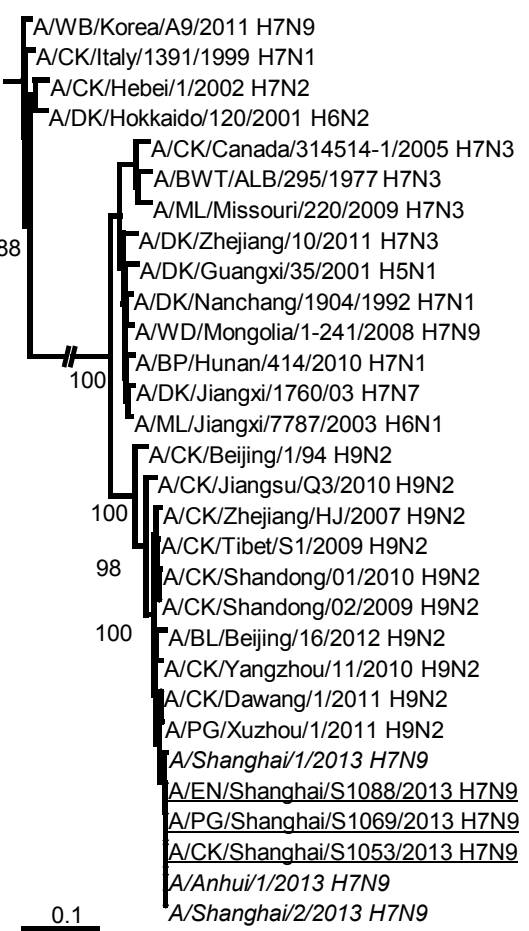

Figure 2 Phylogenetic analyses of PB2 (a), PB1 (b), PA (c), NP (d), M (e) and NS (f) genes of the novel H7N9 influenza viruses. Phylogenetic analysis was performed using ClustalX 1.81 software, implementing the neighbor joining method. Tree topology was evaluated by 1000 bootstrap analyses and shown using Tree View 1.6.6. Neighbor-joining bootstrap values $\geqslant 70$ were shown at the major nodes of the phylogenetic trees. The trees of PB2, PB1, PA, $\mathrm{NP}$ and $\mathrm{M}$ were rooted to A/EQ/Prague/1/56 (H7N7) and the NS tree to A/GS/Guangdong/1/96 (H5N1). The regions of the nucleotide sequences used for the phylogenetic analysis were: PB2, 58-2283; PB1, 48-2230; PA, 25-2029; NP, 46-1523; M, 26-784; NS, 27-719. Viral genes sequenced in this study were underlined in phylogenetic trees. The PB2, PB1, PA, NP, M and NS sequences for the H7N9 viruses causing human infections were downloaded from the database of Global Initiative on Sharing All Influenza Data (GISAID) and were shown in italic. The abbreviations used are the same as those defined in Figure 1. 
live poultry markets of Shanghai when human infections occurred in Shanghai and Anhui Province, China. H7N9 viruses were only isolated from live poultry markets and not from poultry farms, indicating that the former might be the origin of the emergence of the novel H7N9 viruses. Live poultry markets could bring together a number of bird and poultry species from different sources in a high-density setting, thus providing an ideal environment for reassortment among avian influenza viruses of different subtypes.

The novel H7N9 viruses in the present study derived their six internal genes from the avian H9N2 viruses. Their HA genes are most closely related to duck H7N3 viruses isolated from Zhejiang Province in 2011, but share only $\sim 95 \%$ homology. Due to this limited similarity, the origin of the HA gene of the novel H7N9 viruses remains unclear. With respect to the NA gene, several H4N9 and H11N9 viruses obtained from duck or duck farm environmental samples during our active surveillance in Dongting Lake, China, showed considerable similarity to the NA gene of the novel H7N9 viruses. However, whether these Dongting Lake avian influenza viruses are the direct ancestor of the NA gene of the novel H7N9 influenza viruses remains unclear. The only certainty we have is that the novel H7N9 viruses are the product of gene reassortment with the six internal genes derived from one donor and the HA and NA genes from one or two other donors.

Several molecular signatures predicted to be associated with the adaptation of avian influenza viruses to humans were seen in these novel H7N9 viruses. The 226L mutation, a critical residue in the receptor-binding sites of the HA protein, was already present in the avian and environment H7N9 viruses, which may have facilitated infection and replication of the novel H7N9 viruses in humans. Importantly, the three human H7N9 viruses all acquired the $627 \mathrm{~K}$ mutation in their PB2 protein during their replication in human hosts, which is in clear contrast with the avian and environmental H7N9 viruses. This suggests that the novel H7N9 viruses can adapt well to humans. In fact, the adaptability of the H7N9 viruses appears to be superior to that of the H5N1 influenza viruses, which seem to acquire PB2 $627 \mathrm{~K}$ less frequently than the novel H7N9 viruses.

In past decades, $\mathrm{H} 7$ avian influenza viruses have caused large outbreaks in domestic poultry worldwide, resulting in severe economic losses for the poultry industry. During circulation in poultry, $\mathrm{H7}$ influenza viruses can be directly transmitted from poultry to humans and cause disease [20-24]. One example is the highly pathogenic H7N7 influenza virus that was prevalent in domestic poultry in The Netherlands in 2003 [25]. This outbreak resulted in the direct transmission of H7N7 influenza viruses to at least 89 people, with symptoms of conjunctivitis or a mild influenza-like illness and one death [26]. Furthermore, the circulation of low pathogenic $\mathrm{H} 7$ influenza virus in poultry could result in the emergence of a highly pathogenic influenza virus via the acquisition of multiple basic amino acids at the
HA cleavage site, which has occurred with H7N1 influenza viruses during outbreaks in chickens and turkeys in Italy [27]. Currently, the novel H7N9 influenza viruses lack the multiple basic amino acids at the HA cleavage site but are already considerably pathogenic to humans. It will be disastrous if these viruses acquire the multiple basic amino acids during their continued circulation in poultry and humans.

The novel features that the new H7N9 viruses possess, including: previously unidentified HA and NA composition; wide prevalence in avian hosts and the environment; an exceptional adaptive ability in humans; and the potential to acquire multiple basic amino acids at the HA cleavage site and evolve into a highly pathogenic form, are a major cause for concern with respect to public health worldwide. Forceful measures, such as continued surveillance in avian and human hosts, control of animal movement, shutdown of live poultry markets and culling of poultry in affected areas must be taken during this initial stage of virus prevalence to prevent a possible pandemic. It is also imperative to evaluate the pathogenicity and transmissibility of these H7N9 viruses, and to develop effective vaccines and antiviral drugs to combat them and reduce, if not eliminate, their threat to human health.

This work was supported by the National Basic Research Program of China, the China Agricultural Research System (CARS-42-G08), and the National Science and Technology Major Project (2012ZX10004214). We thank Dr. Shu Yuelong at the Chinese National Influenza Center, Chinese Center for Disease Control and Prevention for sharing with us the gene sequence information of the human A (H7N9) influenza viruses.

1 Li C, Hatta M, Watanabe S, et al. Compatibility among polymerase subunit proteins is a restricting factor in reassortment between equine H7N7 and human H3N2 influenza viruses. J Virol, 2008, 82: 1188011888

2 Holland J, Spindler K, Horodyski F, et al. Rapid evolution of RNA genomes. Science, 1982, 215: 1577-1585

3 Yang H, Qiao C, Tang X, et al. Human infection from avian-like influenza A (H1N1) viruses in pigs, China. Emerg Infect Dis, 2012, 18: 1144-1146

4 Hoffmann E, Stech J, Guan Y, et al. Universal primer set for the full-length amplification of all influenza A viruses. Arch Virol, 2001, 146: 2275-2289

5 Senne D A, Panigrahy B, Kawaoka Y, et al. Survey of the hemagglutinin (HA) cleavage site sequence of $\mathrm{H} 5$ and $\mathrm{H} 7$ avian influenza viruses: Amino acid sequence at the HA cleavage site as a marker of pathogenicity potential. Avian Dis, 1996, 40: 425-437

6 Subbarao K, Chen H, Swayne D, et al. Evaluation of a genetically modified reassortant H5N1 influenza a virus vaccine candidate generated by plasmid-based reverse genetics. Virology, 2003, 305: 192200

7 Zhang Y, Zhang Q, Gao Y, et al. Key molecular factors in hemagglutinin and PB2 contribute to efficient transmission of the $2009 \mathrm{H} 1 \mathrm{~N} 1$ pandemic influenza virus. J Virol, 2012, 86: 9666-9674

8 Chen Z, Zhou H, Kim L, et al. The receptor binding specificity of the live attenuated influenza $\mathrm{H} 2$ and $\mathrm{H} 6$ vaccine viruses contributes to vaccine immunogenicity and protection in ferrets. J Virol, 2012, 86: 2780-2786

9 Naeve C W, Hinshaw V S, Webster R G. Mutations in the hemagglutinin receptor-binding site can change the biological properties of an influenza virus. J Virol, 1984, 51: 567-569 
10 Srinivasan K, Raman R, Jayaraman A, et al. Quantitative description of glycan-receptor binding of influenza A virus $\mathrm{H} 7$ hemagglutinin. PLoS One, 2013, 8: e49597

11 Subbarao E K, London W, Murphy B R. A single amino acid in the PB2 gene of influenza A virus is a determinant of host range. J Virol, 1993, 67: 1761-1764

12 Li Z, Chen H, Jiao P, et al. Molecular basis of replication of duck H5N1 influenza viruses in a mammalian mouse model. J Virol, 2005, 79: 12058-12064

13 Gao Y, Zhang Y, Shinya K, et al. Identification of amino acids in HA and $\mathrm{PB} 2$ critical for the transmission of $\mathrm{H} 5 \mathrm{~N} 1$ avian influenza viruses in a mammalian host. PLoS Pathog, 2009, 5: e1000709

14 Hatta M, Gao P, Halfmann P, et al. Molecular basis for high virulence of Hongkong H5N1 influenza A viruses. Science, 2001, 293 : 1840-1842

15 Jackson D, Hossain M J, Hickman D, et al. A new influenza virus virulence determinant: The NS1 protein four C-terminal residues modulate pathogenicity. Proc Natl Acad Sci USA, 2008, 105: 4381-4386

16 Conenello G M, Zamarin D, Perrone L A, et al. A single mutation in the PB1-F2 of H5N1 (HK/97) and 1918 influenza A viruses contributes to increased virulence. PLoS Pathog, 2007, 3: 1414-1421

17 Zamarin D, Ortigoza M B, Palese P. Influenza A virus PB1-F2 protein contributes to viral pathogenesis in mice. J Virol, 2006, 80: 7976-7983

18 Saito R, Sakai T, Sato I, et al. Frequency of amantadine-resistant influenza A viruses during two seasons featuring cocirculation of H1N1 and H3N2. J Clin Microbiol, 2003, 41: 2164-2165
19 Shiraishi K, Mitamura K, Sakai-Tagawa Y, et al. High frequency of resistant viruses harboring different mutations in amantadine-treated children with influenza. J Infect Dis, 2003, 188: 57-61

20 Kurtz J, Manvell R J, Banks J. Avian influenza virus isolated from a woman with conjunctivitis. Lancet, 1996, 348: 901-902

21 Campbell C H, Webster R G, Breese S S Jr. Fowl plague virus from man. J Infect Dis, 1970, 122: 513-516

22 Banks J, Speidel E, Alexander D J. Characterisation of an avian influenza A virus isolated from a human-Is an intermediate host necessary for the emergence of pandemic influenza viruses? Arch Virol, 1998, 143: 781-787

23 Taylor H R, Turner A J. A case report of fowl plague keratoconjunctivitis. Br J Ophthalmol, 1977, 61: 86-88

24 Tweed S A, Skowronski D M, David S T, et al. Human illness from avian influenza H7N3, British Columbia. Emerg Infect Dis, 2004, 10: 2196-2199

25 Koopmans M, Wilbrink B, Conyn M, et al. Transmission of H7N7 avian influenza A virus to human beings during a large outbreak in commercial poultry farms in the Netherlands. Lancet, 2004, 363: 587-593

26 Fouchier R A, Schneeberger P M, Rozendaal F W, et al. Avian influenza A virus (H7N7) associated with human conjunctivitis and a fatal case of acute respiratory distress syndrome. Proc Natl Acad Sci USA, 2004, 101: 1356-1361

27 Capua I, Mutinelli F, Marangon S, et al. H7N1 avian influenza in Italy (1999 to 2000) in intensively reared chickens and turkeys. Avian Pathol, 2000, 29: 537-543

Open Access This article is distributed under the terms of the Creative Commons Attribution License which permits any use, distribution, and reproduction in any medium, provided the original author(s) and source are credited. 